\title{
Studies on the Nature of Antihemophilic Factor (Factor VIII)
}

\author{
FURTHER EVIDENCE RELATING THE AHF-LIKE \\ ANTIGENS IN NORMAL AND HEMOPHILIC PLASMAS
}

\author{
Bruce Bennett, Walter B. Forman, and Oscar D. Ratnofy \\ From the Department of Medicine, Case Western Reserve University, School \\ of Medicine, University Hospitals of Cleveland, and Cleveland Veterans \\ Administration Hospital, Cleveland, Ohio 44106
}

A B S T R A C T Normal human antihemophilic factor (AHF, factor VIII) and the protein antigenically related to it in hemophilic plasma both appeared in the void volume of columns of agarose (Sepharose 4B) during purification of these agents. On ultracentrifugation upon sucrose gradients, both agents had sedimentation characteristics similar to those of an S30 marker. After reduction, the polypeptide chains of purified normal AHF and of the nonfunctional agent from hemophilic patients had an apparent molecular weight of 200,000 as determined by sodium dodecyl sulfate polyacrylamide gel electrophoresis. These observations suggest that AHF, purified as described, exists as a large molecule with subunits of molecular weight of approximately 200,000.

Antisera to normal AHF and the nonfunctional agent from hemophilic plasma appeared to be directed against antigens of similar electrophoretic mobility and precipitating characteristics, present in normal and hemophilic plasma but deficient in severe von Willebrand's disease plasma. Both antisera neutralized the AHF clot-promoting activity present in normal plasma, and this property was removed by absorption of the antisera with concentrates of normal or hemophilic plasma but to a greatly reduced extent by concentrates of von Willebrand's disease plasma. These findings suggest that the antigen detected in normal plasma by the antisera appears on a molecule participating in the AHF clotpromoting reaction.

Dr. Bennett is a Research Fellow of the Pink Family Foundation.

Dr. Ratnoff is a Career Investigator of The American Heart Association.

Received for publication 3 January 1973 and in revised form 26 March 1973.

\section{INTRODUCTION}

Classic hemophilia is characterized by the functional deficiency in the plasma of an agent participating in the intrinsic pathway of blood coagulation, antihemophilic factor (AHF, factor VIII). Recent studies, employing an antiserum raised in rabbits to highly purified preparations of normal human $\mathrm{AHF}$, have indicated that the plasma of hemophilic subjects contains normal or increased quantities of an antigen precipitating with this antiserum, whereas that of patients with von Willebrand's disease has quantities of this antigen reduced in proportion to the reduction of AHF clot-promoting activity (1-5). The antisera used in our earlier studies had the following characteristics: on immunodiffusion they produced a line of identity between hemophilic and normal plasma or purified AHF prepared from normal plasma; on immunoelectrophoresis they produced single precipitin lines of similar mobility with concentrates of normal or hemophilic plasma, and they inhibited the clot-promoting activity of normal plasma in specific assays of AHF activity. Absorption with a fraction rich in AHF prepared from normal plasma or a similar fraction prepared from hemophilic plasma neutralized the inhibitory properties of the antiserum against specific AHF clot-promoting activity (1).

These earlier studies suggest strongly that the precipitin in the antiserum is directed against the AHF molecule itself and that patients with classic hemophilia produce a functionally incompetent but antigenically normal form of AHF. Conceivably, this incompetent AHF is an aberrant form of the molecule or a precursor of the active form of $\mathrm{AHF}$, with which it shares an antigenic site. Alternately, it could be argued that the precipitin detects a molecule distinct from AHF but in- 
separable from it by the methods used. If this hypothesis is correct, the antiserum must contain a second, nonprecipitating antibody which neutralizes the clot-promoting activity of AHF. Such would also necessarily be the case if the precipitin was directed only against a precursor to the functional form of AHF. The fact that concentrates of hemophilic plasma can neutralize the inhibitory effect of the antisera on normal AHF clotpromoting activity, however, appears incompatible with the suggestion that the antibody is directed against a precursor alone and not the functional $\mathrm{AHF}$ molecule.

The present study was undertaken to investigate further the relationship of normal antihemophilic factor and the antigenic but nonfunctional protein present in hemophilic plasma. The observations supply further evidence for the similarity of the material detected as antigen in hemophilic plasma and normal AHF. They support the view that the precipitin demonstrable in antisera directed against human AHF is closely related to the antibody that inactivates the functional activity of $\mathrm{AHF}$.

\section{METHODS}

The techniques used for the preparation of human plasma, crude ethanol concentrates of AHF, purified normal AHF, absorbed antibody against purified AHF, and pooled normal human plasma and for the assay of functional AHF activity have been described (1).

Purification of the nonfunctional antigenic protein from the plasma of individual patients with severe classic hemophilia containing no detectable functional AHF was performed by applying the same techniques to hemophilic plasma that were employed to prepared highly purified normal AHF from normal plasma. At $-3^{\circ} \mathrm{C}$, sufficient $53.3 \%$ ethanol was added to approximately $120-\mathrm{ml}$ samples of hemophilic plasma to bring the final concentration of ethanol to $3 \%$. The mixture was stirred for $30 \mathrm{~min}$ at $-3^{\circ} \mathrm{C}$, and the precipitate was separated at $16,000 \mathrm{~g}$ for 10 $\min$ at $0^{\circ} \mathrm{C}$. The supernatant fluid was discarded, and the precipitate was dissolved in $25 \mathrm{ml}$ imidazole-saline buffer (0.02 M imidazole, $0.14 \mathrm{M} \mathrm{NaCl}, \mathrm{pH} 6.5)$ at room temperature. $25 \mathrm{ml}$ of $20 \%$ (wt/vol) polyethylene glycol (polyethylene glycol 6,000, average molecular weight 6,000-7,500; Matheson, Coleman \& Bell, East Rutherford, N. J.) in imidazole-saline buffer was added at room temperature. The mixture was divided into four aliquots, and the precipitate was separated at $12,000 \mathrm{~g}$ for $10 \mathrm{~min}$. Each precipitate was washed by suspending in $5 \mathrm{ml}$ of cold $6 \%$ ethanol and recentrifuged at room temperature, and the supernatant fluid was discarded. Each precipitate was then dissolved in $2.0 \mathrm{ml}$ imidazole-saline buffer at room temperature with gentle stirring, and the aliquots were combined and resedimented at room temperature for $10 \mathrm{~min}$ to remove any remaining particulate material. The dissolved precipitates were pooled, and this concentrate was then applied to a $5.0 \times 38 \mathrm{~cm}$ column of Sepharose 4B at room temperature and eluted with imidazole-saline buffer at room temperature. employing upward flow. The effluent was collected in $5.0 \mathrm{ml}$ aliquots. Each fraction was assayed for AHF-life clot-promoting activity and for AHF-like antigen. The contents of tubes with highest amounts of antigen were pooled and concentrated to $2.0 \mathrm{ml}$ by pressure dialysis in an Amicon
8MC Micro-ultrafiltration system (Amicon Corp., Lexington, Mass.) using an Amicon PM30 Diaflo membrane. These concentrated preparations contained approximately $0.2 \mathrm{mg}$ protein $/ \mathrm{ml}$. Glycine stabilization of the purified materials was not employed in this study.

Antiserum to purified normal AHF or to the material purified from one hemophilic plasma was raised in New Zealand white female rabbits, and the antiserum was collected as previously described (1). Antiserum to normal AHF was rendered monospecific by absorption with an AHF-poor fraction of normal plasma prepared as previously described (1). The antiserum to hemophilic material was absorbed with a fraction prepared similarly from hemophilic plasma. To remove all procoagulant activity from antisera used in clotting studies these were then incubated at $60^{\circ} \mathrm{C}$ for $60 \mathrm{~min}$, and, after cooling to room temperature, $10 \mathrm{mg}$ tricalcium phosphate was added to each $\mathrm{ml}$ antiserum, the mixture was agitated for $10 \mathrm{~min}$ at room temperature and the serum was separated by centrifugation.

Agarose gels for immunoelectrophoresis and immunodiffusion were prepared as previously described (1). Immunoelectrophoresis was performed on ethanol concentrates using a constant potential of $200 \mathrm{~V}$ for $90 \mathrm{~min}$ at room temperature but otherwise were as previously described, as were immunodiffusion studies.

Quantitative immunoelectrophoresis of $\mathrm{AHF}$ was carried out as previously described (1) in agarose gels each containing $0.05 \mathrm{ml}$ of antiserum to purified normal AHF : crude ethanol concentrates were not employed in this study. $10-\mu 1$ aliquots of the fractions eluted from the columns were applied directly to the wells in the agarose gels; standard curves were made by applying whole plasma in serial dilutions to the wells.

The influence of the antisera on normal AHF was assessed by incubating the antisera with equal volumes of pooled normal human plasma as a source of AHF for $2 \mathrm{~h}$ at $37^{\circ} \mathrm{C}$ and assaying residual AHF activity in the clotpromoting assay. Controls comprised pooled normal plasma incubated with normal rabbit serum, and a control curve was prepared by appropriate dilution of this incubation mixture. On seven separate occasions, this method assessed the inhibitory activity of one antiserum as consistently over $83 \%$ inhibition, of a second antiserum as consistently between 50 and $70 \%$ inhibition, and on three separate occasions that of a third antiserum as between 50 and $60 \%$ inhibition of the control.

Protein concentration was determined by the method of Lowry, Rosebrough, Farr, and Randall (6).

Tests for blocking of the activity of the antisera were performed with concentrates of plasma. Ethanol concentrates were prepared as previously described (1) from 5, 2 , and $1 \mathrm{ml}$ vol of hoth pooled normal plasma and of plasma of a patient with classic hemophilia (who was not the donor of the blood used in preparation of the material used to raise the antiserum). A similar concentrate was prepared from a $5 \mathrm{ml}$ sample of plasma from a patient with severe von Willebrand's disease. Each concentrate was dissolved in $0.7 \mathrm{ml}$ of antiserum that had been diluted in $7 \mathrm{vol}$ of barbital-saline buffer $(0.025 \mathrm{M}$ barbital sodium, $0.125 \mathrm{M} \mathrm{NaCl}, \mathrm{pH} 7.5$ ) and incubated for $2 \mathrm{~h}$ at $37^{\circ} \mathrm{C}$. At the same time, the diluted antiserum was incubated in the same way without addition of ethanol fractions. The mixtures were then incubated for $60 \mathrm{~min}$ at $60^{\circ} \mathrm{C}$ to destroy residual AHF clot-promoting activity present in the concentrates of normal and to a lesser extent in those of von Willebrand's disease plasma. Precipitated material was re- 
moved by centrifuging, and the supernatant fluids were stored overnight at $4^{\circ} \mathrm{C}$. $0.4 \mathrm{ml}$ of each supernatant fraction was mixed with $0.05 \mathrm{ml}$ of pooled normal plasma as a source of $\mathrm{AHF}$ and incubated for $2 \mathrm{~h}$ at $37^{\circ} \mathrm{C}$. $0.55 \mathrm{ml}$ barbital-saline buffer was then added to each mixture, and residual AHF activity was measured without further dilution. AHF activity was compared with that of $0.05 \mathrm{ml}$ pooled normal plasma to hold barbital-saline buffer was added in place of antiserum; standard curves were prepared from these control mixtures, and the amount of inhibition produced by the antiserum absorbed with the various plasma concentrates was estimated by interpolation and compared with that produced by antiserum to which no plasma concentrate had been added. This method in our hands has demonstrated that von Willebrand's disease plasma is less efficient than is normal or hemophilic plasma at blocking antisera to normal human AHF (1). We have also shown that crude correspondence exists between quantities of $\mathrm{AHF}$ detected by clot-promoting or immunoelectrophoresis and this antibody-blocking technique (7).

Purified preparations of AHF from three normal individuals and of the material produced by two patients with hemophilia were subjected to ultracentrifugation by applying $0.3 \mathrm{ml}$ samples to the surface of $5-20 \%$ sucrose gradients in cellulose nitrate cups (Beckman Instruments, Inc., Palo Alto, Calif.) as previously described (8). Subribosomal particles (50S and 30S, General Biochemicals Div., Mogul Corp., Chagrin Falls, Ohio) and porcine thyroglobulin (19S, Sigma Chemical Co., St. Louis, Mo.) were used as markers and were applied to separate gradients. The samples were centrifuged at $39,000 \mathrm{rpm}$ (average, 124,$000 \mathrm{~g}$ ) in an SW39L head of a Beckman Spinco Model L-2 ultracentrifuge at $0^{\circ} \mathrm{C}$ for $3 \mathrm{~h}$. Fractions were collected by puncturing the bottom of each tube. Fractions from tubes containing the test proteins were tested, at a dilution of 1 part in 4 parts of $5 \%$ sucrose, for AHF clot-promoting activity and for AHF-like antigens as described above. Fractions from the tubes containing the marker molecules were tested for protein concentration.

Sodium dodecyl sulfate (SDS) polyacrylamide gel electrophoresis was performed by the technique of Weber and Osborn (9). Purified AHF preparations from three normal subjects and the purified proteins from three hemophilic patients were lyophilized individually after concentration by pressure dialysis. These samples were then incubated for $2 \mathrm{~h}$ at $37^{\circ} \mathrm{C}$ in $0.01 \mathrm{M}$ sodium phosphate buffer, $1 \%$ SDS (Sigma) and 1\% $\beta$-mercaptoethanol (Eastman Kodak Co., Rochester, N. Y.). The protein concentration was $0.1-0.3$ $\mathrm{mg} / \mathrm{ml}$. Aliquots containing $2-5 \mu \mathrm{g}$ of protein from each sample were applied to $5 \%$ polyacrylamide gels containing $1 \%$ SDS and $1 \% \beta$-mercaptoethanol as outlined by Weber and Osborn (9). The glass gel tubes were $8 \mathrm{~cm}$ in length with an internal diameter of $6 \mathrm{~mm}$. Protein standards employed were: bovine pancreatic chymotrypsinogen A $(25,000$ D, Pharmacia Fine Chemicals, Piscataway, N. J.), pepsin (35,000 D, Worthington Biochemical Corp., Freehold, N. J.), human serum albumin $(68,000 \mathrm{D}$, Sigma), Type I bovine thyroglobin ( $160,000 \mathrm{D}$, Sigma), Escherichia coli $\beta$-galactosidase $(135,000 \mathrm{D}$, Sigma $)$, and human transferrin $(88,000$ D, Behringwerke AG, West Germany).

\section{RESULTS}

The peaks of AHF-like clot-promoting activity and of AHF-like antigen separated from concentrates of normal plasma were eluted at the same volume from col-

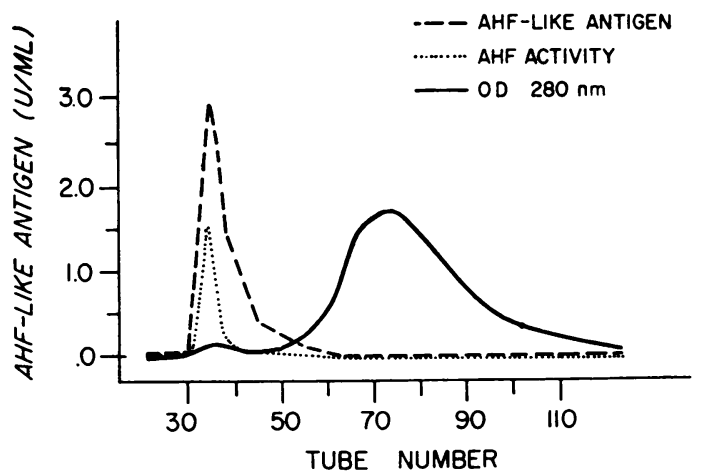

Figcre 1 Representative elution pattern of AHF clotpromoting activity and AHF-like antigen from Sepharose $4 \mathrm{~B}$ column to which a concentrate of normal human plasma was applied.

umns of Sepharose 4B (Fig. 1). Antigenic material from the plasma concentrates of patients with classic hemophilia, detected by quantitative immunoelectrophoresis using antiserum to purified normal AHF, eluted at the same point from the same column (Fig. 2), that is, in the void volume of the column. These results were regularly repeated with normal plasma and were obtained with plasmas from three separate hemophilic patients. Clot-promoting activity was not detected in fractions from runs using hemophilic material. In other experiments, not illustrated, whole plasma samples, rather than ethanol-polyethylene-glycol concentrates of plasma, were applied to a much smaller column $(0.9 \times$ $28 \mathrm{~cm}$ ); under these conditions, too, the antigenic material from hemophilic plasma and the antigenic and clot-promoting agent from normal plasma were eluted in the void volume of the column.

After ultracentrifugation on sucrose density gradients, purified normal AHF activity was found to be maximal

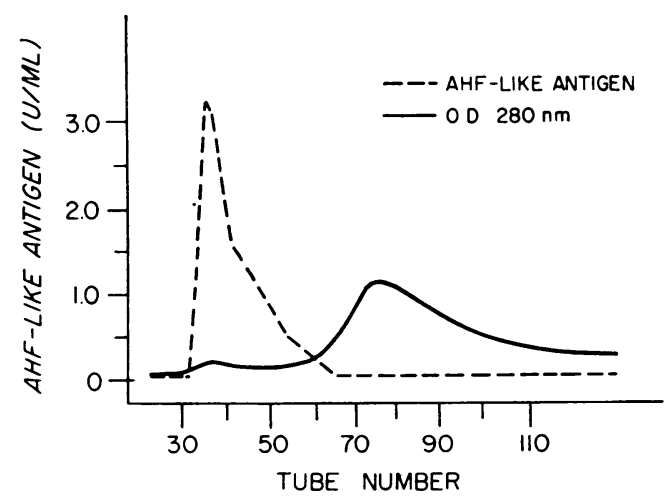

FIGLRE 2 Representative elution pattern of AHF-like antigen from the same column as employed in Fig. 1 to which a concentrate of hemophilic plasma was applied. No clot-promoting activity was detected in the fractions. 


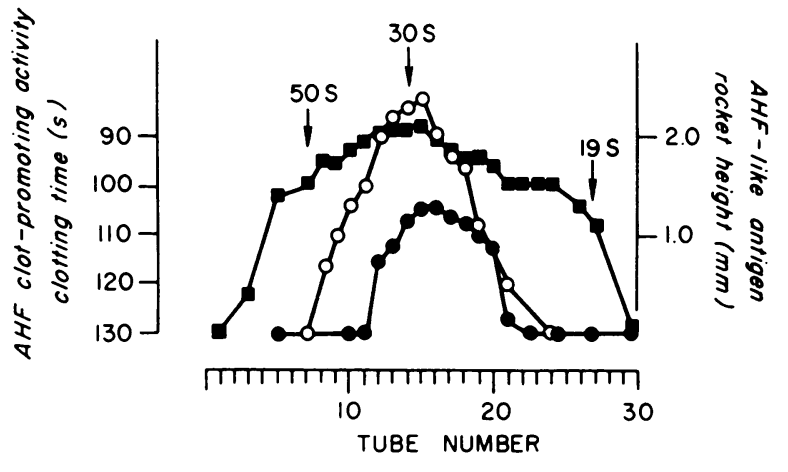

Clot promoting activity in s, Normal AHF

$\longrightarrow$ Antigen - height of rocket, Normal AHF

$\multimap$ Antigen - height of rocket, Hemophilic protein

Figlke 3 Representative sucrose density gradient ultracentrifugation of purified normal $\mathrm{AHF}$ and the purified hemophilic material and of $50 \mathrm{~S}, 30 \mathrm{~S}$, and $19 \mathrm{~S}$ markers. Tube no. 1 was at the bottom of the gradient and tube 30 at the top.

in fractions comparable to those in which $30 \mathrm{~S}$ subribosomal particles were concentrated. Maximal AHF clotpromoting activity coincided with maximal concentration of AHF-like antigen. This result was reproduced 3 times with $\mathrm{AHF}$ prepared from a different normal donor on each occasion. In runs concurrent with these, the antigenic proteins prepared from the plasmas of two hemophilic patients were both concentrated in the same fractions as was the antigenic and clot-promoting agent purified from normal plasma (Fig. 3).

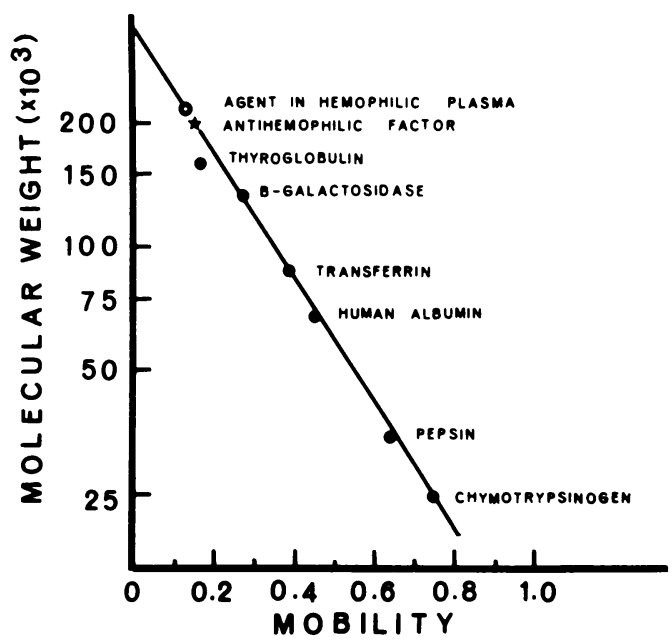

FigLRE 4 Determination of molecular weight by SISSmercaptoethanol acrylamide gel electrophoresis. The extrapolated molecular weight of the fragments are : 215,000 for the agent in hemophilic plasma and 197,000 for antihemophilic factor.
Normal AHF and the agent in hemophilic plasma were reduced with $\beta$-mercaptoethanol and subjected to sodium dodecyl sulfate polyacrylamide electrophoresis. Using this technique, a straight line was obtained when the electrophoretic mobility of marker proteins was plotted against the logarithm of their known polypeptide chain molecular weight. These results were reproducible, varying minimally from experiment to experiment. Under these conditions, after reduction, both normal AHF and the agent in hemophilic plasma had mobilities corresponding to a molecular weight of 200,000 $\pm 10 \%$ (Fig. 4). Three additional trace components were present in variable quantities in each of the three normal and three hemophilic specimens tested.

Incubation of normal plasma with the antiserum to the purified himoplilic protein neutralized its $\mathrm{AHF}$ clot-promoting activity, as determined by specific assays (Table $I$ ). The antisera to hemophilic material appeared to be less powerful inhibitors of AHF than some of the antisera to normal AHF. Whether this represented variability among the rabbits or differences in the antigens cannot be determined at present. Rabbits $\triangle$ and $\mathrm{B}$, which had been immunized with the hemophilic protein, and rabbit D. immunized with normal AHF, had been boosted on only one occasion: antisera from these rabbits inlibited the clot-promoting activity of normal $\mathrm{AHF}$ to similar degrees. In contrast, rabbits

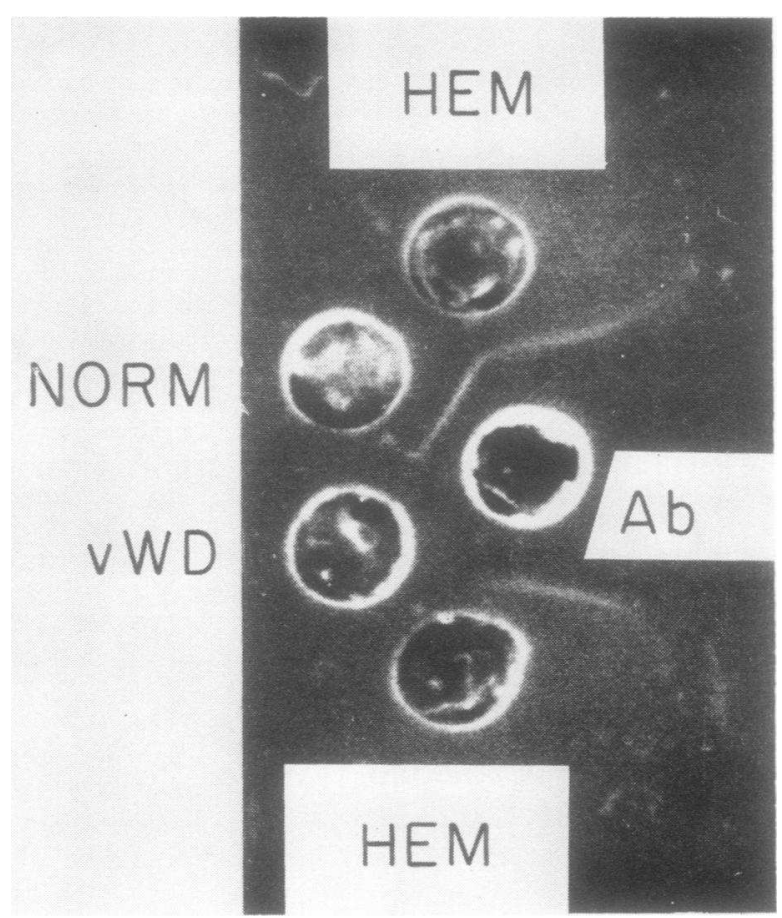

Figure 5 Immunodiffusion of concentrates of normal, hemophilic, and von Willebrand's disease plasma against the rabbit antibody to the hemophilic nonfunctional protein. 
$\mathrm{C}$ and $\mathrm{E}$, immunized with normal AHF, had received several boosting doses of the protein, and the antisera from these animals were more powerful inhibitors of AHF clot-promoting activity.

Concentrates of both normal and hemophilic plasma neutralized the ability of the antisera to inhibit the AHF clot-promoting activity of normal plasma to similar degrees, while concentrates of the plasma of a patient with severe von Willebrand's disease (AHF level 0.1 $\mathrm{U} / \mathrm{ml}$ ) exerted a blocking effect approximately equivalent to only one-fifth of its volume of normal plasma (Table II).

Lines of identity appeared between normal and hemophilic plasma when tested against the antiserum to the hemophilic material (Fig. 5). No precipitin line appeared opposite the well containing a concentrate prepared from plasma of the patient with severe von Willebrand's disease.

The antiserum to the hemophilic material detected antigens with similar mobility in concentrates of normal and hemophilic plasma (Fig. 6a). The antigen detected by this antiserum had identical electrophoretic mobility to the antigen detected by the antiserum against purified normal AHF (Fig. 6b).

\section{DISCUSSION}

The gel filtration studies described indicate that the molecules detected in several normal and hemophilic plasmas by the rabbit antiserum to purified normal AHF have a similar minimal molecular size. They both were found in the void volume of columns of Sepharose 4B when unaltered plasma or concentrates of material pre-
TABLE I

Inhibition of AHF-like Clot-Promoting Acticity by Antisera to Normal AHF and the Hemophilic Material

\begin{tabular}{ccc}
\hline & $\begin{array}{c}\text { Conc. of } \\
\text { incubation } \\
\text { mixture } \\
\text { tested } \\
\text { human plasma }\end{array}$ & $\begin{array}{c}\text { Clotting time } \\
\text { in AHF assay }\end{array}$ \\
\hline Normal rabbit serum & $\%$ & $s$ \\
& 100 & 104.5 \\
Antiserum A & 50 & 114.6 \\
Antiserum B & 25 & 126.5 \\
Antiserum C & 12 & 133.5 \\
Antiserum D & 100 & 117.0 \\
Antiserum E & 100 & 117.5 \\
& 100 & 132.5 \\
& 100 & 115.0 \\
& 100 & 139.0 \\
\hline
\end{tabular}

Antisera $\mathrm{A}$ and $\mathrm{B}$ are those prepared in separate rabbits to the hemophilic material. Antisera C, D, and E are those prepared in separate rabbits to normal AHF. Equal volumes of normal human plasma incubated with normal rabbit serum for $2 \mathrm{~h}$ at $37^{\circ} \mathrm{C}$ and residual $\mathrm{AHF}$ clot-promoting activity assayed. Dilutions containing $100,50,25$, and $12 c^{\circ}$, of this incubation mixture were assayed to provide a control curve. Equal volumes of normal human plasma and the various antisera were incubated similarly and tested without further dilution. It is evident that antisera $\mathrm{A}, \mathrm{B}$, and $\mathrm{D}$ destroyed over $50 \%$ of the AHF clot-promoting activity in the incubation mixtures and antisera $\mathrm{C}$ and $\mathrm{E}$ destroyed approximately $90 \%$ of the AHF clot-promoting activity.

pared by ethanol and polyethylene glycol precipitation of plasma were applied. Thus the hemophilic material has a similar minimal molecular weight to that previ-

TABLE II

Inhibition of $A H F$ Procoagulant Activity Produced by Antiserum which had been Absorbed with Varying Amounts of Ethanol Concentrates of Normal, Hemophilic, or con Willebrand's Disease Plasma (\% Inhibition)

\begin{tabular}{|c|c|c|c|c|c|c|c|c|}
\hline & \multicolumn{8}{|c|}{ Absorbants of antiserum } \\
\hline & \multicolumn{3}{|c|}{ Normal plasma } & \multicolumn{3}{|c|}{ Hemophilic plasma } & \multirow{2}{*}{$\begin{array}{c}\mathrm{vWD} \\
\text { plasma, } \\
5 \mathrm{ml}\end{array}$} & \multirow[b]{2}{*}{ Buffer } \\
\hline & $5 \mathrm{ml}$ & $2 \mathrm{ml}$ & $1 \mathrm{ml}$ & $5 \mathrm{ml}$ & $2 \mathrm{ml}$ & $1 \mathrm{ml}$ & & \\
\hline & & $\%$ & & & $\%$ & & $\%$ & $\%$ \\
\hline Antiserum A to hemophilic protein & 35 & 40 & 58 & 36 & 40 & 55 & 53 & 66 \\
\hline Antiserum $\mathrm{C}$ to normal $\mathrm{AHF}$ & 38 & 55 & 64 & 45 & 55 & 60 & 60 & 70 \\
\hline
\end{tabular}

Antisera to hemophilic protein or to normal AHF absorbed with ethanol concentrates of varying volumes of plasma from normal individuals, and patients with classic hemophilia and von Willebrand's disease. Residual clot-promoting activity was removed and residual capacity to inhibit AHF clot-promoting activity was assayed as described in the Methods section. It is evident that antisera absorbed with buffer only inhibited 66 and $70 \%$ of the AHF employed; those absorbed with concentrates of $1 \mathrm{ml}$ normal or hemophilic plasma had had their inhibitory power reduced to a degree similar to those absorbed with concentrates of $5 \mathrm{ml}$ von Willebrand's disease plasma (i.e. to about $60 \%$ inhibition). Antisera absorbed with concentrates of larger volumes of normal and hemophilic plasma showed progressively greater reduction in their ability to inhibit AHF clot-promoting activity. 

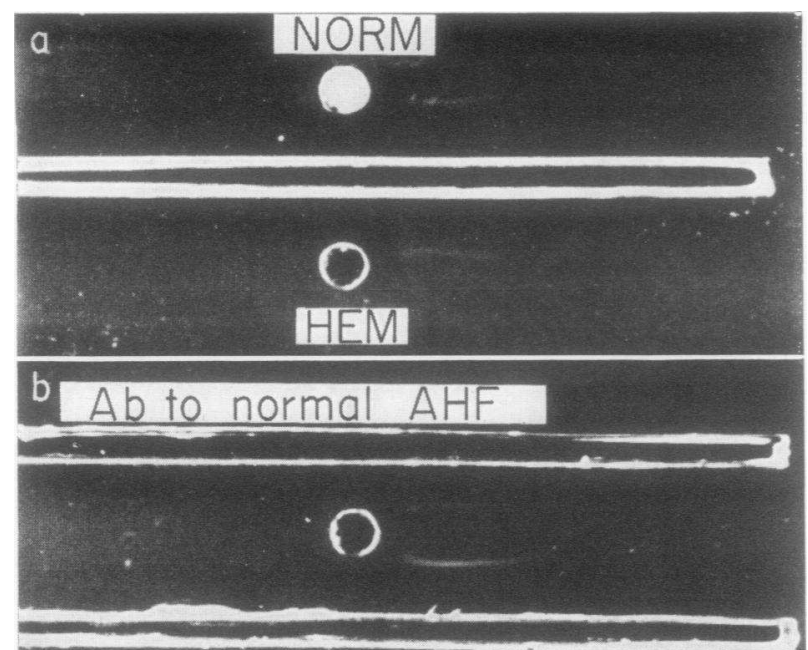

Ab to hemophilic material

FIGLRE 6 Immunoelectrophoretic studies on the antiserum to the nonfunctional hemophilic protein: (a) immunoelectrophoresis of concentrates of normal and hemophilic plasma against the antiserum to the hemophilic material; and $(b)$ immunoelectrophoresis of concentrate of normal plasma against antiserum to normal $\mathrm{AHF}$ and antiserum to the hemophilic material.

ously suggested for normal AHF under these conditions, namely $2 \times 10^{3}(6)$. This prediction assumes that the molecules are spherical and that molecular complexing has not occurred, both of which assumptions may prove to be unjustified. Ultracentrifugal study of partially purified normal human $\mathrm{AHF}$ has alrealy established that the clot-promoting agent is of large dimensions (8). Our observations on normal AHF purified by a different method also indicate that the molecule, under the conditions employed, is of large size, similar to that of $30 \mathrm{~S}$ subribosomal particles and, further. that the nonfunctional agents from hemophilic plasma appears to have similar dimensions.

The results of the sodium dodecyl polyacrylamide gel electrophoretic studies, however, suggest that both the hemophilic protein and normal AHF contained subunits each with a molecular weight of about 200,000. This accords with the suggestion of other workers of a subunit structure for normal AHF and the nonfunctional protein in hemophilic plasma. though the size of the subunits reported has varied widely (10-15). Marchesi, Shulman, and Gralnick (13) observed that treatment of AHF with SDS alone did not result in disaggregation of normal AHF, while fragments with a molecular weight of approximately 240,000 D were separated by reduction with dithioerythritol. Anderson and his associates reported recently that the molecular weight of the reduced subunits was $195.000 \mathrm{D}$ (10).
Earlier, the same group had estimated the molecular weight of the reduced fragments as approximately 300,000 , and similar subunits prepared from hemophilic plasma had approximately the same molecular weight (11). In additional, unpublished experiments, we have found that the subunits of $\mathrm{AHF}$ can be separated by column chromatography of reducted AHF preparations of normal plasma. supporting the riew that the subunits are joined by disulfide bridges.

Antiserum to the material prepared from hemophilic plasma was found to have properties similar to that raised against preparations of normal human AHF. Both antisera neutralized the clot-promoting activity in normal plasma in specific assays for antihemophilic activity, a property that was removed to approximately similar degrees by absorption with concentrates separated from both normal and hemophilic plasma. A concentrate of von Willebrand's disease plasma was much less effective in this respect. Both antisera contained precipitins recognizing antigens in normal and hemophilic plasma which are absent from severe von Willebrand's disease plasma. The antigens detected by both antisera in normal and hemophilic plasma have the same electrophoretic mobility. In effect, the antisera behave as if they detected the same antigen.

The fact that the antiserum to the material prepared from hemophilic plasma neutralized the clot-promoting activity of normal AHF suggests strongly that the antigen it detected is situated on a molecule necessary for the expression of AHF clot-promoting activity. The absence of AHF clot-promoting activity from the hemophilic protein suggests that the area corresponding to the active site of normal AHF is altered in an, as yet, unknown way. It therefore seems unlikely that antiserum to the hemophilic material contained an antibody (precipitating or otherwise) directed against the active site of normal AHF. Destruction of the clot-promoting activity of AHF of normal individuals by the antiserum to the hemophilic material suggests therefore that the antigen recognized by the antiserum lies on the AHF molecule itself but not at the active clot-promoting site. These findings do not preclude the possibility that the antigen may also be present in a precursor of functional AHF in normal individuals, nor do they indicate whether the antigenic material derived from hemophilic plasma represents such a precursor or an abnormal form of the AHF molecule.

\section{ACKNOWLEDGMENTS}

This study was supported in part by research grant HI. 01661 from the National Heart and Lung Institute, the National Institutes of Health, U. S. Public Health Service, and in part by grants from The American Heart Association, the Northeastern Ohio Heart Association, and the Veterans Administration Research Fund-MRIS 2240-01. 


\section{REFERENCES}

1. Zimmerman, T. S., O. D. Ratnoff, and A. E. Powell. 1971. Immunologic differentiation of classic hemophilia (factor VIII deficiency) and von Willebrand's disease. J. Clin. Invest. 50: 244.

2. Meyer, D., J. N. Lavergne, M. J. Larrieu, and F. Josso. 1972. Cross-reacting material in congenital factor VIII deficiencies (haemophilia $\mathrm{A}$ and von Willebrand's disease). Thromb. Res. $1: 183$.

3. Hoyer, L. W. 1972. Immunologic studies of antihemophilic factor (AHF, factor VIII) III. Comparative binding properties of human and rabbit anti-AHF. Blood. 39: 481.

4. Stites, D. P., E. J. Hershgold, J. D. Perlman, and H. H. Fudenberg. 1971. Factor VIII detection by hemagglutination inhibition: hemophilia A and von Willebrand's disease. Science (Wash. D. C.). 171: 196.

5. Bouma, B. N., Y. Wiegerinck, J. J. Sixma, J. A. van Mourik, and I. A. Mochtar. 1972. Immunological characterization of purified anti-haemophilic factor A (factor VIII) which corrects abnormal platelet retention in von Willebrand's disease. Nature (Lond.). 236: 104.

6. Lowry, O. H., N. J. Rosebrough, A. L. Farr, and R. J. Randall. 1951. Protein measurement with the Folin phenol reagent. J. Biol. Chem. 193: 265.

7. Bennett, B., and O. D. Ratnoff. 1972. Changes in antihemophilic factor (AHF, Factor VIII) procoagulant activity and AHF-like antigen in normal pregnancy, and following exercise and pneumoencephalography. $J$. Lab. Clin. Med. 80: 256.

8. Kass, L., O. D. Ratnoff, and M. A. Leon. 1969. Studies on the purification of antihemophilic factor (factor VIII). J. Clin. Invest. 48: 351.

9. Weber, K., and M. Osborn. 1969. The reliability of molecular weight determinations by dodecyl sulfatepolyacrylamide gel electrophoresis. J. Biol. Chem. 244 : 4406.

10. McKee, P. A. 1970. Purification and electrophoretic analysis of human antihemophilic factor (factor VIII). Fed. Proc. 29 : 647. (Abstr.)

11. McKee, P. A., and L. S. Lessin. 1970. Purification and structural studies of human antihemophilic factor (factor VIII). Clin. Res. 18: 80. (Abstr.)

12. Hershgold, E. J. 1971. The subunit structure of human factor VIII (antihemophilic factor). Fed. Proc. 30: 540. (Abstr.)

13. Marchesi, S. L., N. R. Shulman, and H. R. Gralnick. 1972. Studies on the purification and characterization of human factor VIII. J. Clin. Invest. 51: 2151.

14. Anderson, J. C., and P. A. McKee. 1972. Effects of proteolytic enzymes on the coagulant properties and molecular structure of human factor. VIII. Circulation. 46 (Suppl. II) : 52. (Abstr.)

15. Legaz. M., G. Schmer, and E. Davie. 1972. Characterization of human factor VIII (antihemophilic factor). Circulation. 46 (Suppl. II) : 53. (Abstr.) 